03

\title{
Новый способ обработки изображений, полученных шлирен-методом с фоновым экраном
}

\author{
() Ю.А. Баринов \\ Физико-технический институт им. А.Ф. Иофффе РАН, Санкт-Петербург, Россия \\ E-mail: yury@mail.ioffe.ru
}

Поступило в Редакцию 27 февраля 2019г.

В окончательной редакции 27 февраля 2019г.

Принято к публикации 29 марта 2019г.

\begin{abstract}
Проведено тестирование шлирен-метода с фоновым экраном. В качестве объекта использовался поток горячего воздуха с известной температурой. Обнаружено, что на объектах миллиметрового размера метод может давать некорректный результат. Выяснено, что ошибка возникает при использовании стандартного программного обеспечения. Предложены другой способ обработки и его программная реализация.
\end{abstract}

Ключевые слова: шлирен-метод, шлирен-метод с фоновым экраном.

DOI: 10.21883/PJTF.2019.12.47920.17759

Способы визуализации газовых течений, такие как например шлирен-методы, давно применяются в экспериментах $[1,2]$. Методы используются и для количественных измерений, например, такого важного параметра, как температура. При определенных условиях шлирен-методы применимы и при исследовании плазменных объектов [3]. В то же время организация эксперимента и получение количественной информации сопряжены с рядом трудностей. Благодаря развитию цифровых способов регистрации изображений и компьютерной технике появился новый метод, основанный на использовании фонового экрана (background oriented schlieren, BOS) [4]. Отличительные особенности метода - простота реализации и доступное программное обеспечение. BOS-метод применим как для визуализации газовых течений, так и для получения количественной информации. Метод основан на программном обеспечении, которое применяется для определения скорости частиц (particle image velocimetry, PIV) [5]. Здесь представлены ссылки на несколько таких программ: свободная программа PIVlab для коммерческой среды MATLAB [6], свободные программы OpenPIV [7] и JPIV [8] и др. Принцип работы метода основан на сравнении двух изображений. Первое - исходное изображение $(A)$ фонового экрана. Экран заполнен хаотичными или регулярными точками. Второе - измененное изображение $(B)$ того же фонового экрана, которое получается после прохождения света через исследуемый объект. Изменение показателя преломления в исследуемом объекте вызывает изменения изображения, т.е. смещение точек $[9,10]$. Программное обеспечение PIV находит величину смещения точек на измененном изображении $B$ относительно изображения A. Показатель преломления связан с плотностью среды уравнением Лорентца-Лоренца или в более простом виде уравнением Гладстона-Дейла. Если исследуемая среда подчиняется закону идеального газа, то, используя эти уравнения, можно определить температуру. Цель настоящей работы - на примере определения температуры газовых потоков оценить возможности BOS-метода для исследования разряда с жидкими неметаллическими электродами [11,12].

Для проверки BOS-метода использовалась термовоз-

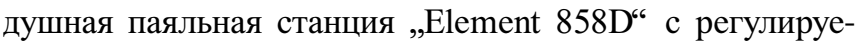
мой температурой потока воздуха $100-500^{\circ} \mathrm{C}$. На фен паяльной станции устанавливалась насадка, формирующая поток воздуха $(\varnothing 4 \mathrm{~mm})$. Поток воздуха можно рассматривать как цилиндрически симметричный объект. На рис. 1, а показана схема эксперимента. Изображение регистрировалось цифровым фотоаппаратом с размером матрицы $4608 \times 3456$ рх. Использовался объектив с фокусным расстоянием $150 \mathrm{~mm}$ и входным диаметром $\sim 30 \mathrm{~mm}$. Расстояние фокусировки для этого объектива $(Z 1+Z 2) \geqslant 900 \mathrm{~mm}$, расстояние от матрицы фотоаппарата до потока воздуха $Z 2=270 \mathrm{~mm}$. На изображении вырезалась область примерно на $4 \mathrm{~mm}$ выше насадки фена, для этой области проводились расчеты температуры. Использовался фоновый экран с регулярным расположением точек. Такой фоновый экран позволяет легко контролировать смещение визуально по фотографии. Величина смещения точек $\Delta x$ определялась с помощью программы PIVlab. Поскольку объект симметричный, и кривая смещения должна быть симметрична относительно оси объекта. Тогда кривую можно разделить на две половинки (левую и правую) и определять температуру раздельно. Результат расчета температуры для потока воздуха $T=200^{\circ} \mathrm{C}$ представлен на рис. $1, b$. Видно, что значения получаются нереалистичными. В процессе поиска ошибки обнаружилось, что алгоритм программного обеспечения PIV не позволяет корректно определить смещение точек. Оценка смещения между видимыми границами точек, выполненная вручную, показала, что ошибка может доходить до $50 \%$. На рис. $2, a$ для примера представлены части изображений $A$ и $B$. Видно, что исходные точки $(1-3)$ фонового экрана $A$ на 


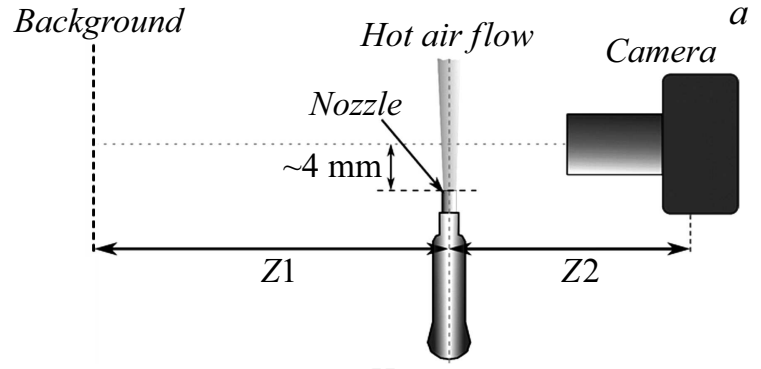

Hot air gun

$b$

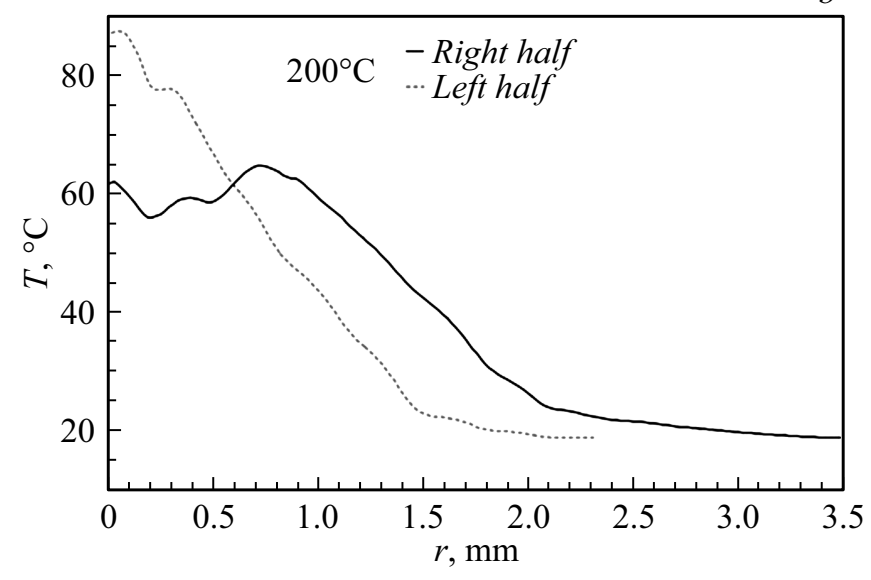

Рис. 1. $a-$ схема эксперимента; $b-$ расчет температуры.

изображении $B$ сильно вытянуты $\left(1^{\prime}-3^{\prime}\right)$. Такие изменения на изображении $B$ вызваны небольшими размерами исследуемого объекта. Действительно, если размер входного диаметра объектива заметно больше размеров объекта, то часть света будет проходить мимо объекта, тем самым формируя неизмененное изображение. В результате на изображение $B$ будет накладываться часть изображения $A$. Так как радиус объекта мал, возможен заметный градиент температуры на размере точки фонового экрана. Из-за этого пиксели ближе к оси объекта сместятся больше, тем самым создавая вытянутое изображение точки в направлении градиента. Процесс обработки усложняется рассеянием света в объекте, что снижает контраст изображения $B$. Полностью устранить эти причины в используемой экспериментальной схеме не представляется возможным. Если входной диаметр объектива еще можно изменить, то свойства объекта изменить нельзя. Сильное уменьшение размера точек экрана не решает проблему. Из-за рассеяния света в объекте точки небольшого размера могут вообще потеряться. Таким образом, изображения объектов с заметным градиентом температуры на масштабе размера точки фонового экрана нельзя обрабатывать в программax PIV. Если говорить упрощенно, то положение точек в этих программах определяется по геометрическому центру точек. Поэтому часть информации о смещении теряется. Анализ причин ошибки позволяет предложить простой способ решения этой проблемы. Можно измерять расстояния между видимыми границами точек (рис. 2,b). В общем случае это нужно делать по двум координатам. Для проверки этой идеи была написана программа в свободной математической среде Scilab [13] с использованием дополнительного пакета для работы с изображениями IPCV [14]. Программа названа „Point Shift Measurement" (PSM), текст программы находится на сайте [15]. В программе PSM изображения $A$ и $B$ последовательно обрабатываются с помощью цифровых фильтров и подпрограмм пакета IPCV. На завершающем этапе по заданной величине порога выделяются границы и вычисляются смещения. Результат сравнения обработки изображений в программах PIVlab и PSM для одной и той же температуры воздуха $200^{\circ} \mathrm{C}$ представлен на рис. 3,a. Видно, что формы кривых заметно разли-

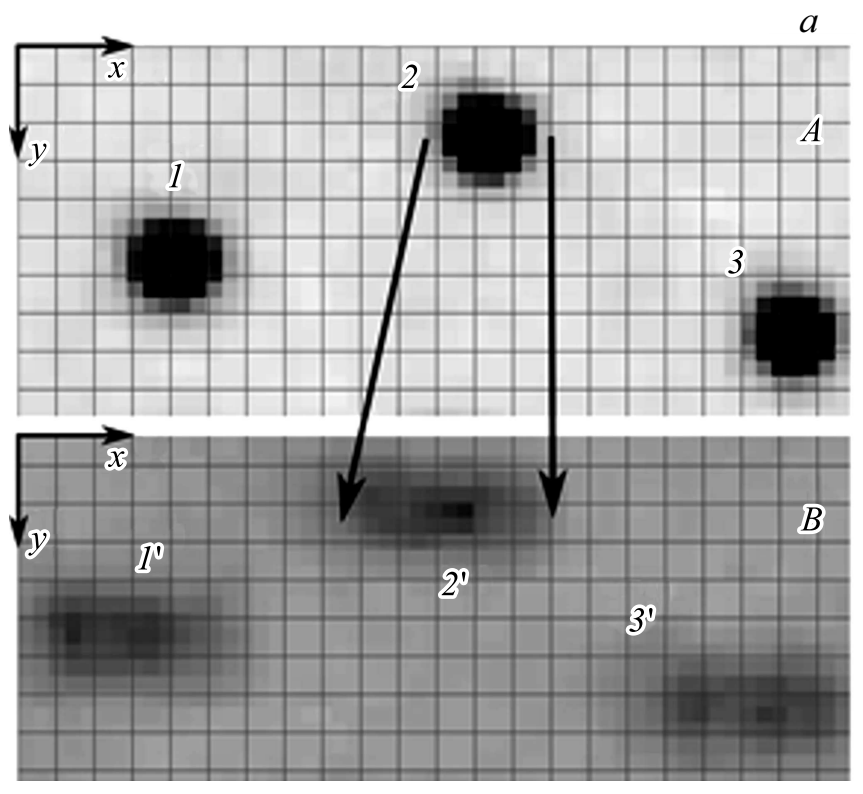

$b$

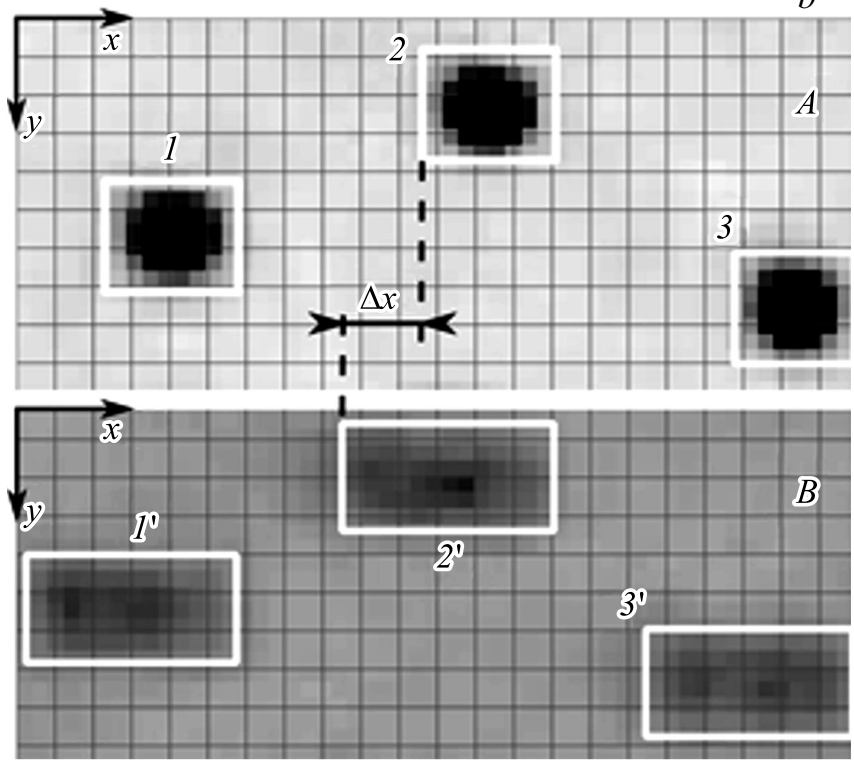

Рис. 2. $a-$ пример части изображений: изображение $A$ (верхняя часть) и изображение $B$ (нижняя часть); $b-$ измерение расстояния между границами. 

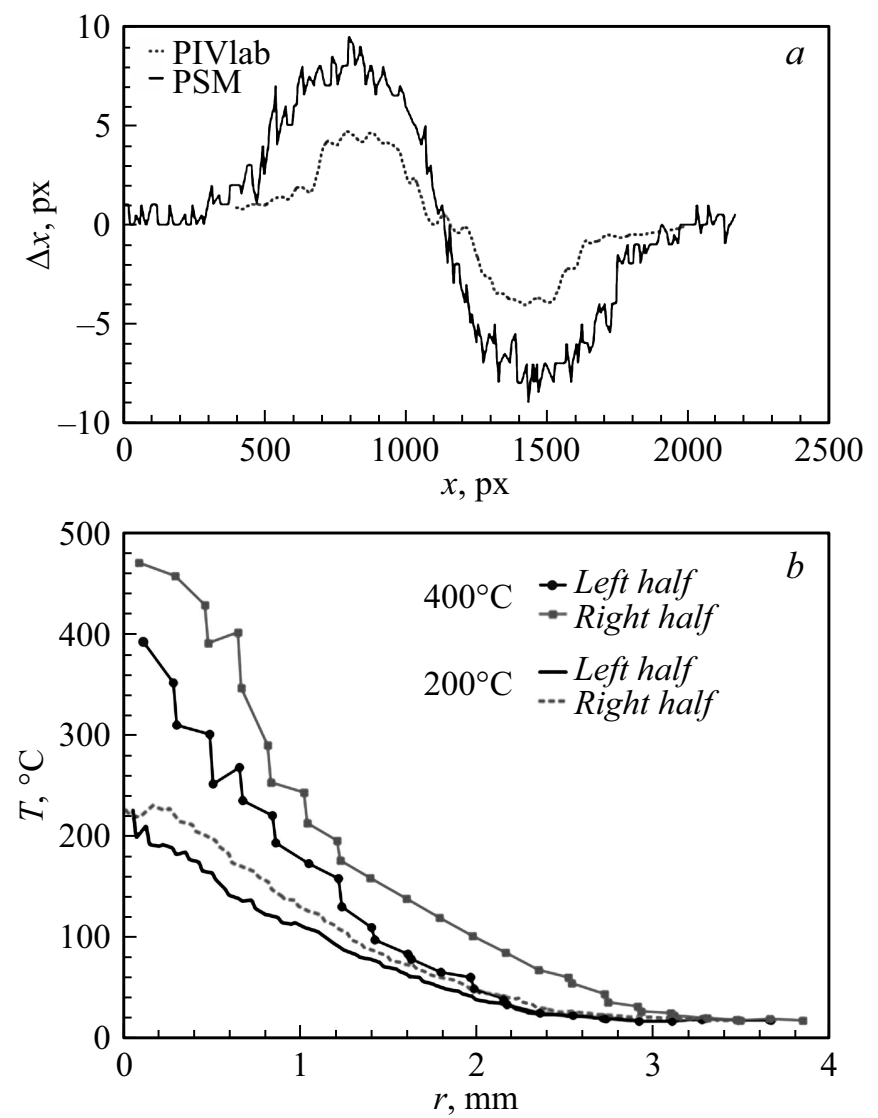

Pис. 3. $a-$ сравнение обработки в программах PIVlab и PSM; $b-$ расчет температуры для потока воздуха 200 и $400^{\circ} \mathrm{C}$.

чаются. Максимальное смещение $\Delta x$ для PSM больше примерно в 2 раза, чем для PIVlab, это совпадает с визуальным наблюдением. Обработка в программе PSM была выполнена для двух температур потока воздуха $\left(200\right.$ и $\left.400^{\circ} \mathrm{C}\right)$, расчет температуры представлен на рис. $3, b$. Полученный результат гораздо лучше согласуется с ожидаемой температурой.

На данный момент программу PSM следует рассматривать как демонстрацию другого способа обработки. Из-за простоты программа имеет ряд ограничений. Количество точек фонового экрана на изображении исследуемой области не должно быть большим, иначе процесс обработки может сильно затянуться. Расстояние между точками фонового экрана должно быть больше ожидаемого смещения, это принципиальное ограничение. Для получения лучшего результата программу необходимо усовершенствовать, но даже в таком виде обработка изображений получается удовлетворительной. Точность определения температуры можно улучшить набором большого количества данных с последующим усреднением, а также сглаживанием исходных данных. Как видно, программу можно использовать при исследовании газовых потоков малого размера. В плазменных объектах градиенты температуры могут быть более сильными, поэтому для диагностики плазменных объек- тов могут потребоваться дополнительные изменения в коде программы. Данный метод определения смещения можно реализовать с помощью других программных сред, позволяющих обрабатывать изображения и обнаруживать объекты на них (как, например, библиотека OpenCV [16]).

\section{Список литературы}

[1] Васильев Л.А. Теневые методы. М.: Наука, 1968. 400 с.

[2] Merzkirch $W$. Flow visualization. 2nd ed. Orlando: Academic Press, 1987. $260 \mathrm{p}$.

[3] Hermoch V. // Czech. J. Phys. 1970. V. 20. N 8. P. 939-949.

[4] Meier G. // Exp. Fluids. 2002. V. 33. N 1. P. 181-187. DOI: $10.1007 / \mathrm{s} 00348-002-0450-7$

[5] Raffel M., Willert C.E., Scarano F., Kähler C.J., Wereley S.T., Kompenhans $J$. Particle image velocimetry: a practical guide. 2nd ed. Heidelberg-N.Y.: Springer, 2007. 448 p.

[6] Thielicke W., Stamhuis E.J. // J. Open Res. Software. 2014. V. 2. N 1. P. e30. DOI: 10.5334 /jors.bl

[7] Taylor Z.J., Gurka R., Kopp G., Liberzon A. // IEEE Trans. Instrum. Meas. 2010. V. 59. N 12. P. 3262-3269. DOI: 10.1109/TIM.2010.2047149

[8] Vennemann P. JPIV [Электронный pecypc]. URL: http://www.jpiv.vennemann-online.de/index.html (дата обращения: 05.02.2019).

[9] Goldhahn E., Seume J. // Exp. Fluids. 2007. V. 43. N 2-3. P. 241-249. DOI: 10.1007/s00348-007-0331-1

[10] Raffel M. // Exp. Fluids. 2015. V. 56. N 3. P. 60. DOI: $10.1007 / \mathrm{s} 00348-015-1927-5$

[11] André P., Barinov Yu., Faure G., Shkol'nik S. // J. Phys. D: Appl. Phys. 2018. V. 51. N 44. P. 445202. DOI: $10.1088 / 1361-6463 /$ aadfad

[12] Баринов Ю.А., Школьник С.М. // ЖТФ. 2016. Т. 86. В. 11. C. $155-158$. DOI: $10.21883 /$ jtf.2016.11.43833.1833

[13] Scilab.org [Электронный pecypc]. URL: https://www.scilab.org/ (дата обращения: 05.02.2019).

[14] Scilab Module: Image Processing and Computer Vision Toolbox [Электронный ресурc].

URL: https://atoms.scilab.org/toolboxes/IPCV/ (дата обращения: 05.02.2019).

[15] Barinov J. Point Shift Measurement (PSM) [Электронный pecypc]. URL: https://github.com/JurySpb/PSM-scilab (дата обращения: 05.02.2019).

[16] Bradski G. // Dr. Dobb's J. Software Tools. 2000. V. 25. N 11. P. $120-125$. 